\title{
Nutraceutical agents with anti-inflammatory properties prevent dietary saturated-fat induced disturbances in blood-brain barrier function in wild-type mice
}

Ryusuke Takechi ${ }^{1,2}$, Menuka M Pallebage-Gamarallage ${ }^{1}$, Virginie Lam ${ }^{1}$, Corey Giles ${ }^{1}$ and John C Mamo ${ }^{1,2^{*}}$

\begin{abstract}
Background: Emerging evidence suggests that disturbances in the blood-brain barrier (BBB) may be pivotal to the pathogenesis and pathology of vascular-based neurodegenerative disorders. Studies suggest that heightened systemic and central inflammations are associated with BBB dysfunction. This study investigated the effect of the anti-inflammatory nutraceuticals garlic extract-aged (GEA), alpha lipoic acid (ALA), niacin, and nicotinamide (NA) in a murine dietary-induced model of BBB dysfunction.

Methods: C57BL/6 mice were fed a diet enriched in saturated fatty acids (SFA, 40\% fat of total energy) for nine months to induce systemic inflammation and BBB disturbances. Nutraceutical treatment groups included the provision of either GEA, ALA, niacin or NA in the positive control SFA-group and in low-fat fed controls. Brain parenchymal extravasation of plasma derived immunoglobulin $\mathrm{G}(\mathrm{lgG})$ and large macromolecules (apolipoprotein (apo) B lipoproteins) measured by quantitative immunofluorescent microscopy, were used as markers of disturbed BBB integrity. Parenchymal glial fibrillar acidic protein (GFAP) and cyclooxygenase-2 (COX-2) were considered in the context of surrogate markers of neurovascular inflammation and oxidative stress. Total anti-oxidant status and glutathione reductase activity were determined in plasma.
\end{abstract}

Results: Brain parenchymal abundance of IgG and apoB lipoproteins was markedly exaggerated in mice maintained on the SFA diet concomitant with significantly increased GFAP and COX-2, and reduced systemic antioxidative status. The nutraceutical GEA, ALA, niacin, and NA completely prevented the SFA-induced disturbances of $\mathrm{BBB}$ and normalized the measures of neurovascular inflammation and oxidative stress.

Conclusions: The anti-inflammatory nutraceutical agents GEA, ALA, niacin, or NA are potent inhibitors of dietary fat-induced disturbances of BBB induced by systemic inflammations.

Keywords: Garlic extract-aged, Alpha-lipoic acid, Blood-brain barrier, Inflammation, Neurodegenerative disorders, Niacin, Nicotinamide, Oxidative stress, Saturated fatty acids

\footnotetext{
* Correspondence: j.mamo@curtin.edu.au

${ }^{1}$ School of Public Health, Curtin Health Innovation Research Institute,

Biosciences Research Precinct, Faculty of Health Sciences, Curtin University, Kent st, Bentley 6102WA, Australia

${ }^{2}$ Centre for Metabolic Fitness, Australian Technology Network, GPO Box U1987, Perth 6845WA, Australia
} 


\section{Background}

Accumulating evidence suggests that disturbances in cerebral capillary integrity characterized by inflammation, loss of blood-brain barrier (BBB) functions, parenchymal extravasation of plasma proteins, proteinaceous deposits on extracellular matrices, and glial cell activation contribute to the onset or progression of a number of neurodegenerative disorders including vascular dementia, Alzheimer's disease, Parkinson's disease, and multiple sclerosis [1]. Cerebral capillary abnormalities that precede frank pathological or clinical abnormalities include capillary endothelial cell proliferation, perivascular gliosis, and progressive arteriopathy, and thereafter the formation of lacunar lesions [2-5].

Cerebral capillary dysfunction induced as a consequence of a chronically heightened state of systemic inflammation is positively associated with neurovascular degenerative disorders [6-9]. Comorbidities such as dyslipidemia, hypertension or endocrine disorders, exposure to pollutants such as smoking, alcohol consumption, or atherogenic diets may also increase neurodegenerative disease onset via a cerebral capillary axis [3,6,8-10]. Studies in animal models support the latter. Wild-type mice chronically fed pro-inflammatory diets enriched in saturated fatty acids (SFA) or cholesterol showed hallmark features of BBB dysfunction including cerebral extravasation of large plasma proteins (immunoglobulin G (IgG) and macromolecules (apolipoprotein (apo) B lipoproteins)) concomitant with diminished endothelial tight junction proteins [9,11-13].

Several in vivo and in vitro studies suggest that pharmacological agents with anti-inflammatory or anti-oxidative activity may positively regulate $\mathrm{BBB}$ integrity through regulation of systemic inflammatory pathways [14-16]. The potent antioxidant, probucol, preserved BBB function and profoundly attenuated astroglial cell activation in SFA-fed mice [17]. The cholesterol-lowering 3-hydroxymethyl-Co-A reductase inhibitor, atorvastatin, prevented $\mathrm{BBB}$ disturbances in spontaneously hypertensive rats [18]. Atorvastatin, pravastatin, and the non-selective cyclooxygenase inhibitor, ibuprofen, were shown to also restore $\mathrm{BBB}$ function in mice with $\mathrm{BBB}$ dysfunction induced by chronic ingestion of a pro-atherogenic diet [18].

Bioactive anti-inflammatories may also confer benefit to cerebral capillary vessels [19,20]. A bitter melonattenuated dietary-fat induced BBB disturbances in wildtype mice concomitant with a significant reduction in both central and systemic inflammatory/oxidative stress markers [7]. However, paradoxical observations have been reported in other studies. Provision of omega-3 fatty acids to mice that had been maintained on an SFA enriched diet for twelve weeks, exacerbated measures of BBB dysfunction [11]. A study by Mustata et al. also showed increased glycoxidation in tendon, aorta, and plasma by anti-oxidative green tea and vitamins $\mathrm{C}$ and $\mathrm{E}$ in a diabetic rat model [21]. Soy isoflavones, such as genistein, inhibit the lipid peroxidation only with superphysiological concentrations due to its poor peroxyl radical scavenging function [22].

In the current study, we investigated the efficacy of selected anti-oxidative nutraceuticals, namely garlic extract-aged (GEA), alpha-lipoic acid (ALA), niacin, and nicotinamide (NA), in an established dietary-induced model of BBB disruption. The GEA contains anti-oxidative phytochemicals including $\mathrm{S}$-allylcysteine, S-allylmercaptocysteine, diallyl sulfide, and allicin, which can scavenge reactive oxygen species. The GEA was shown to inhibit lipid peroxidation and reduce expression of key inflammatory proteins such as nuclear factor-kappa $\mathrm{B}$ in vivo and in vitro [23-25]. In clinical studies, GEA supplementation was found to reduce measures of oxidative stress and showed therapeutic benefits in subjects with Parkinson's and Alzheimer's disease [26-31]. ALA is a naturally occurring anti-oxidant that sequesters free radicals. ALA acts indirectly to enhance cellular anti-oxidant status by stimulating uptake of exogenous anti-oxidants and enhancing the production of endogenous anti-oxidant enzymes. ALA also inhibits the production of proinflammatory cytokines [32]. In a traumatic brain injury model, Toklu et al. reported that ALA improved BBB function concomitant with attenuated central and systemic inflammation [33]. Niacin and NA are classified within the B3 group of vitamins; however, niacin also has significant lipid-lowering effects [34]. Both niacin and NA can be converted into nicotinamide adenine dinucleotide or nicotinamide adenine dinucleotide phosphate, key mediators in enzymatic anti-oxidative reactions [34]. Morris et al. reported that niacin retards the cognitive decline in Alzheimer's disease and, consistent with those findings, NA was found to delay the cognitive decline in Alzheimer's subjects and reduce amyloid deposition in amyloid transgenic mice [35].

\section{Materials and methods}

\section{Animals and diets}

Wild-type C57BL/6J female mice were purchased from Animal Resources Centre, WA, Australia. The low-fat (LF) control chow was the maintenance diet (AIN-93M, Specialty Feeds, WA, Australia) containing < $4 \%(\mathrm{w} / \mathrm{w})$ polyunsaturated fats and was free of SFA or dietary cholesterol. Mice with dietary-induced BBB dysfunction were fed a semi-synthetic diet enriched in SFA (SF07-050, Specialty Feeds, WA, Australia) containing 40\% energy of fat derived from cocoa butter as previously described (5\% w/w palmitic 16:0, 7\% stearic 18:0) [17]. The nutraceutical supplements GEA (Kyolic, USA), ALA, niacin, or NA (all Sigma-Aldrich, USA) were incorporated into either the LF or SFA diets at a concentration of $3 \%$ 
(w/w), $0.2 \%(\mathrm{w} / \mathrm{w}), 1 \%(\mathrm{w} / \mathrm{w})$, or $0.3 \%(\mathrm{w} / \mathrm{w})$, respectively. The dose of nutraceutical ingested relative to weight was within clinical recommendations and similar to previous studies [23,26-28,30-35]. The components of GEA are standardized by the manufacturer relative to the amount of S-allylcysteine [36]. Animals were kept in individually ventilated cages with $12 \mathrm{~h}$ light/dark cycle, under controlled temperature $\left(21^{\circ} \mathrm{C}\right)$ and air pressure. All animals had ad libitum access to the diets and water. All the procedures described in this study were approved by NHMRC accredited Curtin Animal Ethics Committee (approval no. N34-10).

\section{Dietary/nutraceutical intervention and sample collection}

Eight mice were randomly allocated at six weeks of age to dietary or nutraceutical treatment groups of LF control chow; LF diet supplemented with GEA (LF+GEA); LF diet supplemented with ALA (LF+ALA); LF diet supplemented with niacin $(\mathrm{LF}+\mathrm{Niacin})$; LF diet supplemented with NA $(\mathrm{LF}+\mathrm{NA})$; diet enriched in SFA; SFA diet supplemented with GEA (SFA+GEA); SFA diet supplemented with ALA (SFA+ALA); SFA diet supplemented with niacin (SFA+ Niacin); or SFA diet supplemented with NA (SFA+NA). After nine months of dietary/nutraceutical intervention, mice were anesthetized with pentobarbital $(45 \mathrm{mg} / \mathrm{kg}$ ). Blood samples were collected from a cardiac puncture, and plasma was isolated and stored at $-80^{\circ} \mathrm{C}$ until next use. The brain was carefully removed and fixed in $4 \%$ paraformaldehyde (w/v in PBS, pH 7.2) for $24 \mathrm{~h}$. Following cryoprotection with $20 \%$ sucrose for $72 \mathrm{~h}$ at $4^{\circ} \mathrm{C}$, the brain tissues were frozen in dry ice/isopentane and stored at $-80^{\circ} \mathrm{C}$ until next use.

\section{Assessment of BBB integrity}

The integrity of the cerebrovascular BBB was assessed by an established method of cerebral plasma protein extravasation that indicates the unspecific blood-tobrain leakage of plasma proteins and macromolecules $[9,11,12,17,18,37,38]$. Briefly, 3-D quantitative immunomicroscopy was used to assess brain parenchymal abundance of IgG (Mw $155 \mathrm{kDa}$ ) and of apoB lipoproteins (molecular weight $>2 \times 10^{7} \mathrm{kDa}$ ). Briefly, $20 \mu \mathrm{m}$ thick cryosections were prepared from the right hemisphere of frozen brain tissues. After blocking with $10 \%$ goat serum for $30 \mathrm{~min}$, the sections were incubated with goat antimouse IgG conjugated to Alexa488 (1:200, Invitrogen, US) for $20 \mathrm{~h}$ at $4^{\circ} \mathrm{C}$. For the immunodetection of apoB, the sections were incubated with rabbit anti-mouse apoB (1:400, Abcam) for $20 \mathrm{~h}$ at $4^{\circ} \mathrm{C}$, followed by an incubation with anti-rabbit IgG secondary antibody conjugated to Alexa488 (1:200, Invitrogen) for $2 \mathrm{~h}$ at $20^{\circ} \mathrm{C}$. Following DAPI nuclei counterstaining, the sections were observed under a fluorescent microscope (Axiovert 200M, Zeiss, Germany). A positive control of BBB dysfunction from mice euthanized with carbon dioxide was utilized.

\section{Immunomicroscopic detection of cerebral inflammation}

Neuronal inflammation was assessed by determining the abundance of parenchymal glial-fibrillar acidic protein (GFAP) and cyclo-oxygenase-2 (COX-2) using a 3-D quantitative immunofluorescent method previously described [12,17]. Briefly, $20 \mu \mathrm{m}$ thick cryosections of the right brain hemisphere were prepared. Non-specific biding sites were blocked with $10 \%$ goat serum in PBS for $30 \mathrm{~min}$ at $20^{\circ} \mathrm{C}$. Either rabbit anti-mouse GFAP (1:200, Abcam, UK) or rabbit anti-mouse COX-2 (1:200, Abcam) was applied to the sections for $20 \mathrm{~h}$ at $4^{\circ} \mathrm{C}$. The sections were then incubated with goat anti-rabbit IgG conjugated with Alexa488 (1:200, Invitrogen) for $2 \mathrm{~h}$ at $20^{\circ} \mathrm{C}$. DAPI was used to counterstain the nuclei.

\section{3-D quantitative immunomicroscopy}

Immunofluorescent micrographs were quantitatively analyzed in a 3-D context as previously described [12,17]. One section per animal was used in the estimated stereotaxic areas of $1.7 \mathrm{~mm}$ interaural and $-2.1 \mathrm{~mm}$ Bregma. Immunofluorescent micrographs were captured with mRM digital camera (Zeiss) attached to Axiovert 200M. At a magnification of $200 \times(20 \times$ Zeiss PlanNeofluar objective with $10 \times$ mRM camera), a minimum of five and three 3-D images was captured from randomly selected areas of the cortex and hippocampal formation, respectively, utilizing the AxioVision imaging software (Zeiss). The random images capture process represents approximately half of the cortex and hippocampal formation region of each section. Each 3-D image consisted of 12 Z-stack 2-D images, and the distance between the Z-stack images were $1.225 \mu \mathrm{m}$

Table 1 Mean weight and weight gain

\begin{tabular}{lllllllllll}
\hline & LF & LF+GEA & LF+ALA & LF+Niacin & LF+NA & SFA & SFA+GEA & SFA+ALA & SFA+Niacin & SFA+NA \\
\hline Final mean weight (g) & 23.36 & 25.01 & 21.71 & 22.81 & 23.16 & 23.56 & 24.95 & $20.51^{*}$ & 23.16 & 22.13 \\
SEM & 0.3995 & 0.7448 & 0.2806 & 0.6906 & 0.5106 & 1.154 & 0.4088 & 0.4228 & 0.2275 & 0.4956 \\
Mean weight gain (g) & 9.05 & 9.26 & 5.26 & 7.78 & 7.27 & 8.25 & 9.76 & 4.85 & 7.96 & 7.29
\end{tabular}

The mean weights with standard error of mean at the end of nine months dietary intervention are shown in low-fat (LF), saturated fat (SFA) or diets supplemented with nutraceutical aged garlic extract-aged (+GEA), alpha-lipoic acid (+ALA), niacin (+Niacin), or nicotinamide (+NA) groups. The differences of means from the beginning to the end of dietary intervention in each group are also shown. *Indicates the statistical significance at $P<0.05$ compared to SFA group by using one-way ANOVA followed by Tukey's post hoc test. 

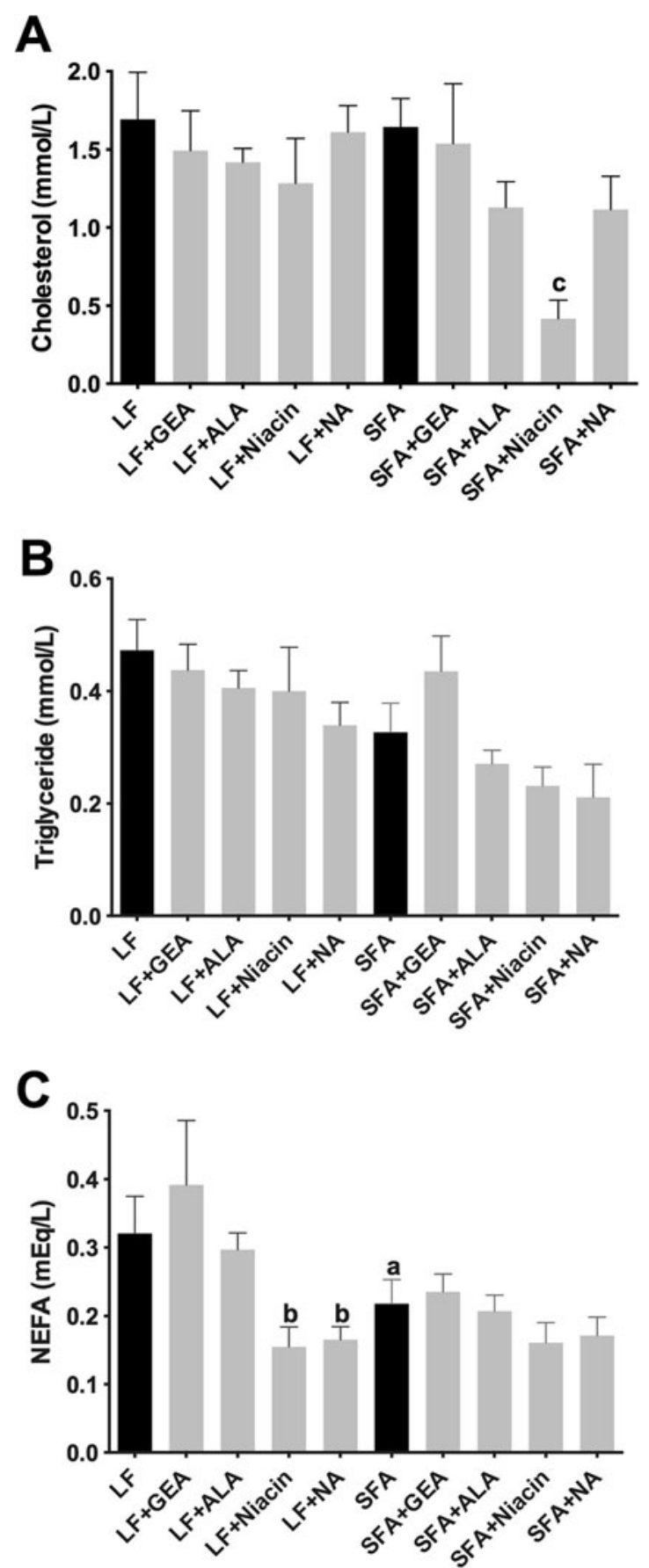

Figure 1 Plasma lipids. The plasma concentrations of cholesterol (A), triglyceride (B), and non-esterified fatty acid (NEFA) (C) were analyzed with commercially available colorimetric kits in mice maintained on low-fat control chow (LF), diet enriched in saturated fat (SFA) or each diet supplemented with nutraceutical garlic extract-aged (+GEA), alpha-lipoic acid (+ALA), niacin (+Niacin) or nicotinamide (+NA) for nine months. One-way ANOVA followed by Tukey's post hoc test was used to analyze the statistical significance at $P<0.05$. (a) Significant difference between LF and SFA; (b) Significant difference between LF and each nutraceutical treatment; and (c) Significant difference between SFA and each nutraceutical treatment. optimized based on Nyquist overlap theory. The voxel intensity of fluorescent dye was then analyzed in 3-D with Volocity 6.2 image analysis software (PerkinElmer, UK). Means of total fluorescent intensities of all the images in cortex and hippocampal formation regions were calculated within each animal, and thereafter compared between the groups.

\section{Plasma oxidative markers}

Plasma anti-oxidative status was assessed by determining the plasma concentration of glutathione reductase and plasma total anti-oxidant status (TAS) using commercially available colorimetric assays (Randox, UK). The details are as described in the manufacturer's instructions but with minor modifications. Briefly, for the glutathione reductase assay, $4 \mu \mathrm{L}$ of plasma sample was mixed with $100 \mu \mathrm{L}$ of substrate buffer and incubated at $37^{\circ} \mathrm{C}$. Exactly at $1,2,3,4$, and 5 min after adding $20 \mu \mathrm{L}$ of NADPH solution, the optical absorbance was read at $340 \mathrm{~nm}$. By plotting the absorbance values, the slope of the linear portion of the curve was determined. The glutathione reductase activity was calculated by determining the reaction rate at $340 \mathrm{~nm}$ with the NADPH extinction coefficient of $0.00622 / \mu \mathrm{M} / \mathrm{cm}$.

The TAS assay measures the anti-oxidative status of the samples by measuring the suppression of radical cation 2,2'-azino-di-[3-ethylbenzthiazoline sulphonate] (ABTS) production. Briefly, $2 \mu \mathrm{L}$ of water, standard or samples were incubated with $100 \mu \mathrm{L}$ of chromogen solution at $37^{\circ} \mathrm{C}$, and the absorbance was read at $600 \mathrm{~nm}$ (A1). At exactly $3 \mathrm{~min}$ after adding $20 \mu \mathrm{L}$ of substrate buffer, the absorbance was read at $600 \mathrm{~nm}$ (A2). The suppression of ABTS formation (A2 - A1) was determined with the known value of the standard (2.14 $\mathrm{mmol} / \mathrm{L})$.

\section{Plasma lipids}

Commercially available colorimetric assays were used to measure the concentrations of plasma cholesterol, triglycerides (Randox, UK), and non-esterified fatty acids (NEFA) (WAKO, Japan) according to the manufacturer's instruction with some minor modifications.

Briefly, for cholesterol and triglyceride assays, $2 \mu \mathrm{L}$ of plasma samples or standards were loaded to a 96 well microplate; $200 \mu \mathrm{L}$ of reaction solution was then added and incubated for $5 \mathrm{~min}$ at $37^{\circ} \mathrm{C}$. The optical absorbance was read at $550 \mathrm{~nm}$. For the NEFA assay, $7 \mu \mathrm{L}$ of plasma samples or standards were loaded to a 96-well microplate; $300 \mu \mathrm{L}$ of Reagent 1 was added and incubated for $3 \mathrm{~min}$ at $37^{\circ} \mathrm{C}$, then $150 \mu \mathrm{L}$ of Reagent 2 was added for $4.5 \mathrm{~min}$ at $37^{\circ} \mathrm{C}$. The optical absorbance was read at $550 \mathrm{~nm}$. 

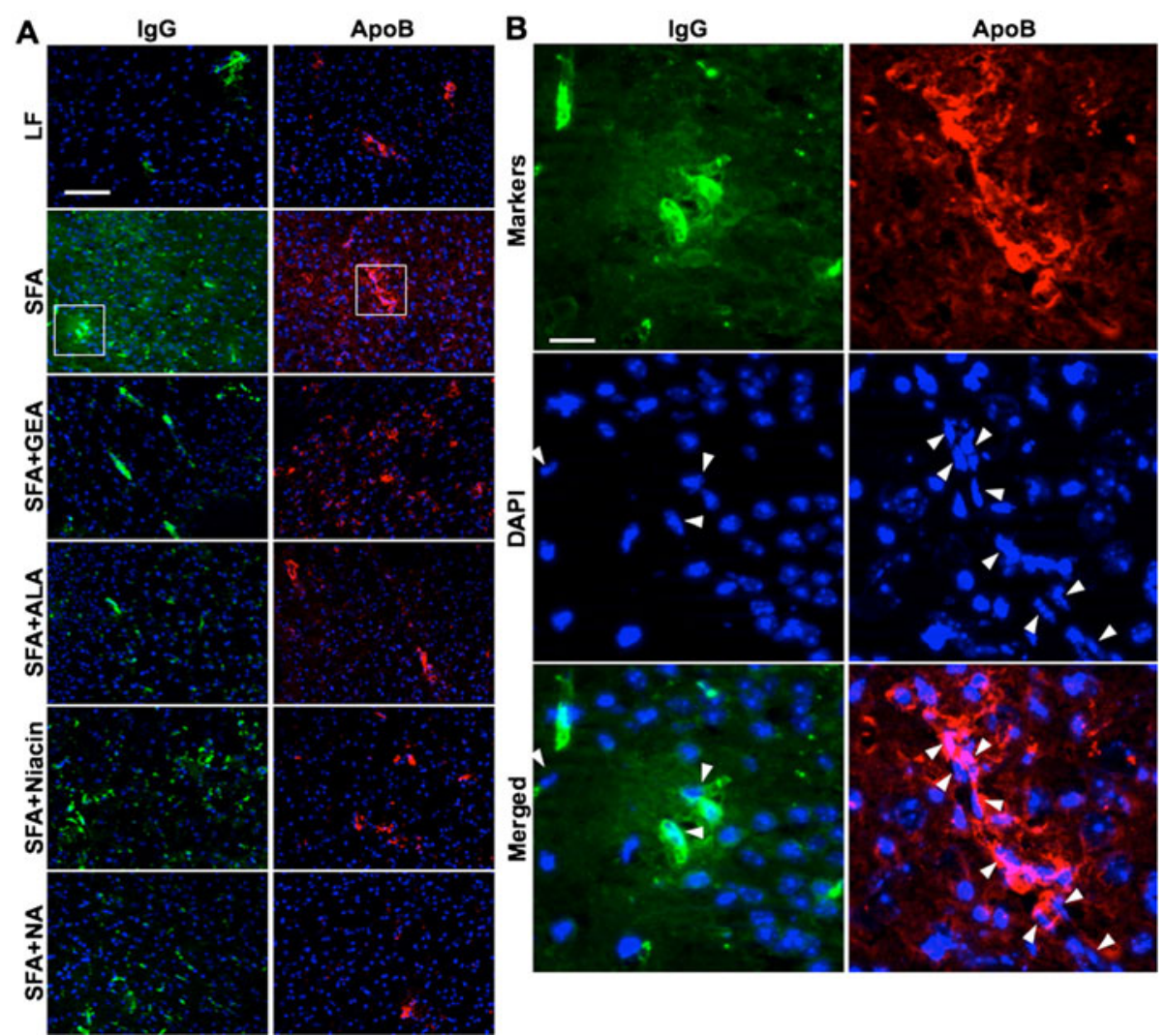

Figure 2 Immunofluorescent micrographs of cerebral extravasations of plasma IgG and apolipoprotein B lipoproteins. The integrity of the BBB was assessed by the detection of brain parenchymal extravasation of plasma derived IgG, and apoB lipoproteins in mice maintained on low-fat control chow (LF), diet enriched in saturated fat (SFA) or each diet supplemented with nutraceutical garlic extract-aged (+GEA),

alpha-lipoic acid (+ALA), niacin (+Niacin), or nicotinamide (+NA). (A) The representative images of cerebral extravasation of IgG or apoB in the cortex are shown in green or red, respectively. Nuclei are shown in blue. Scale bar indicates $100 \mu \mathrm{m}$. (B) The magnified images of the regions of interest are indicated by a white rectangle in frame A. Vascular endothelial cells are shown with white arrowheads. Scale bar indicates $12 \mu \mathrm{m}$.

\section{Statistical analysis}

Each dietary and nutraceutical intervention group contained a minimum of eight mice, which was predicted to provide sufficient power based on the coefficient of variation of the key measures previously published $[9,11,12,17,18]$. All the statistical analyses were conducted by one-way ANOVA analyses followed by Tukey's post hoc tests. The Pearson's correlation coefficient describing the association between parenchymal protein extravasation (IgG and apoB) and parenchymal inflammatory markers, or plasma oxidative markers was determined. Statistical significance was considered at $P<0.05$. Data were expressed as means \pm SEM.

\section{Results}

Mice have similar weight gain pattern and plasma lipids Animals maintained on the diets enriched in SFA and diets supplemented with nutraceutical agents were well tolerated. The total calories consumed were approximately equivalent for the mice maintained on LF control chow and high SFA diet, and a similar weight gain was recorded in most treatment groups for a nine-month intervention period (Table 1). However, the final mean weight of the SFA+ALA group of mice was less than the SFA group $(P<0.05)$.

The plasma concentration of cholesterol and triglyceride of mice maintained on an SFA diet was not significantly different from that of mice maintained on an LF diet (Figure 1A and B). However, the plasma concentration of NEFA was significantly reduced in SFA fed mice compared to LF fed mice $(P<0.05)$ (Figure $1 C)$. Supplementation of niacin in SFA diet significantly reduced plasma cholesterol, whereas no difference was found in LF+niacin group. Both niacin and NA supplementation significantly lowered the plasma NEFA concentration in the LF mice.

Chronic ingestion of SFA diet causes severe breakdown of $B B B$, but BBB integrity is restored by nutraceutical agents We confirm substantial parenchymal perivascular abundance of plasma-derived proteins (IgG) and macromolecules (apoB lipoproteins) in mice maintained on SFA 
A

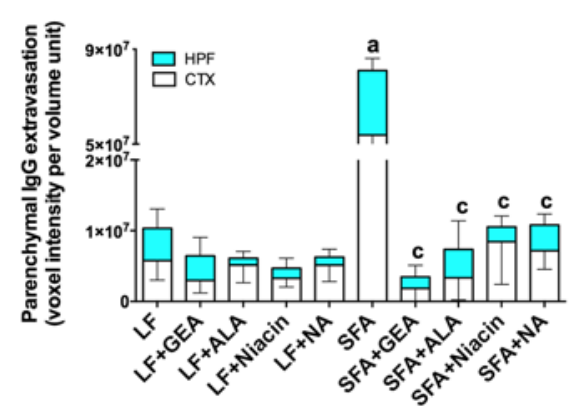

B

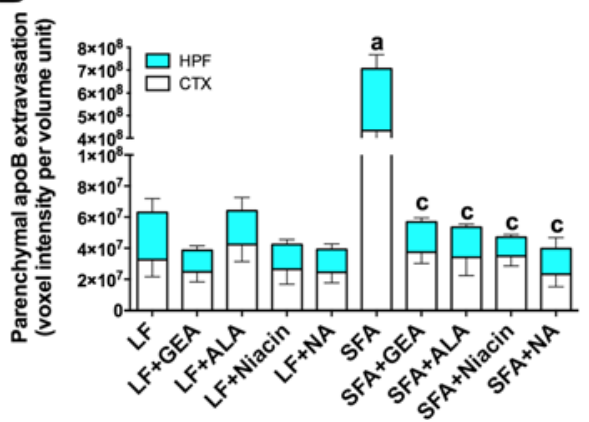

Figure 3 3-D quantitative analyses of cerebral IgG and apoB extravasation. The optical staining intensities of $\lg G(\mathbf{A})$ and apoB $(\mathbf{B})$ were measured with 3-D image analysis software and shown in the cortex (CTX) and hippocampal formation (HPF) of mice maintained on low-fat control chow (LF), diet enriched in saturated fat (SFA) or each diet supplemented with nutraceutical garlic extract-aged (+GEA), alpha-lipoic acid (+ALA), niacin (+Niacin), or nicotinamide (+NA) for nine months. One-way ANOVA followed by Tukey's post hoc test was used to analyze the statistical significance at $P<0.05$. Data were analyzed in cortex and hippocampal formation separately, however shown as combined since the statistical significance was identical. (a) Significant difference between LF and SFA; (c) Significant difference between SFA and each

nutraceutical treatment.

enriched diet for nine months (Figure 2). The 3-D quantitative immunomicroscopy showed that the parenchymal extravasation of IgG and apoB are substantially greater in SFA fed mice compared to mice maintained on LF control chow (Figure 3). However, provision of GEA, ALA, niacin, or NA with the SFA diet completely ameliorated the BBB damaging effect of dietary-SFA (Figures 2 and 3). The control mice maintained on LF diet for nine months showed some moderate signs of cerebral IgG and apoB lipoprotein extravasation (Figure 2 and 3), however this effect was not attenuated by cosupplementation with GEA, ALA, niacin, or NA.

\section{Parenchymal inflammations occur following the BBB disruption}

Evidence of significant neurovascular inflammation and oxidative stress was concomitant with compromised BBB integrity in mice maintained on an SFA enriched diet for nine months. Cerebral parenchymal abundance of GFAP was elevated 3-fold in SFA mice compared to LF-fed mice at nine-months (Figure 4). Similarly, COX-2 expression was substantially increased because of chronic ingestion of the SFA-enriched chow (Figure 4). Consistent with the suppression of parenchymal plasma protein extravasation in SFA mice, provision of either GEA, ALA, niacin, or NA, completely suppressed the SFA-induced expression of GFAP and COX-2 (Figures 4 and 5A,D). A heightened state of neurovascular inflammation was not indicated by GFAP and COX-2 in mice maintained on the LF-diet for nine months (Figures 4 and $5 \mathrm{~A}, \mathrm{D})$. A causal association between inflammation (indicated by GFAP and COX-2 abundance) and BBBdysfunction (parenchymal IgG and apoB lipoprotein abundance) is suggested by Pearson's correlation analysis (Figure 5B,C,E,F).
Nutraceutical agents restore the systemic oxidative status Plasma total anti-oxidative status was expressed relative to in vitro suppression of enzymatic genesis of a cation radical. Plasma TAS was significantly less in SFA fed mice compared to the LF fed control (Figure 6A). In contrast, mice supplemented with either GEA, ALA, niacin, or NA in the SFA treatment groups had a plasma TAS similar to age-matched low-fat controls. Pearson's correlation analysis showed that the plasma TAS was negatively associated with parenchymal IgG and apoB lipoprotein extravasation in mice (Figure 6B,C). Glutathione reductase activity was not reduced in SFA mice compared to controls and provision of nutraceuticals had no significant effect (Figure 6D). Glutathione reductase activity was not associated with cerebral IgG or apoB-lipoprotein extravasation (Figure 6E,F).

\section{Discussion}

The present study investigated the efficacy of the antioxidant nutraceuticals GEA, ALA, niacin, and NA in preventing BBB disruption in genetically unmanipulated mice maintained on a pro-inflammatory diet enriched in SFA. Consistent with previous studies showing progressive deterioration of $\mathrm{BBB}$ commensurate with the duration of feeding $[7,9,11,12,17,18]$, in this study mice maintained on the SFA diet for nine months had substantial breakdown of BBB function. Compromised BBB integrity was indicated by established methodologies which considered the parenchymal abundance of plasma derived IgG, and also by the extravasation of larger macromolecules (apoB triglyceride rich lipoprotein) $[9,11,12,17,18,37,38]$. Quantitative 3-D immunohistochemical microscopy indicated that cerebral extravasation of IgG and apoB were substantially greater in the mice maintained on the SFA diet for nine months 


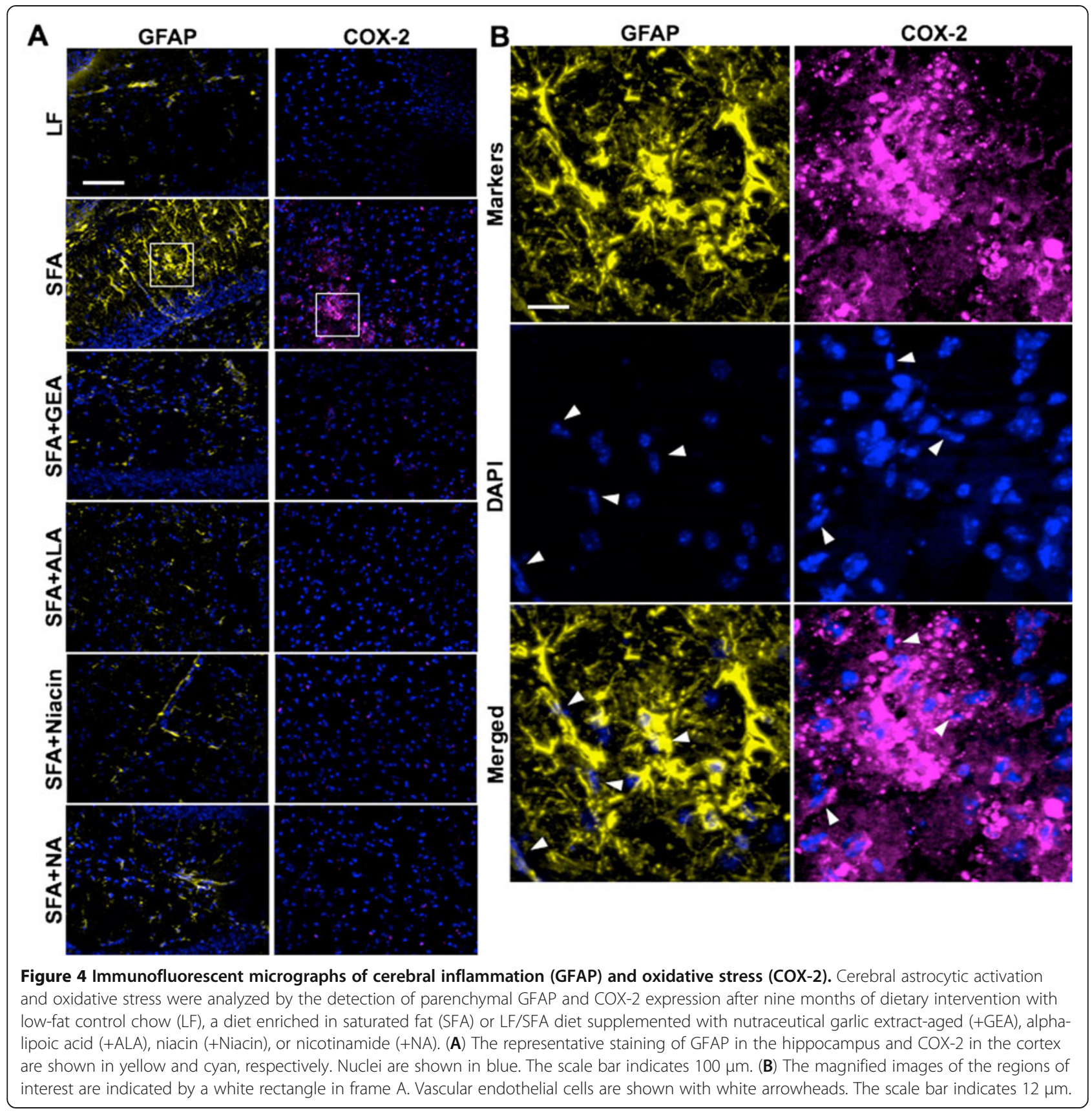

compared to the control LF-fed mice. Moreover, the dietary SFA-induced blood-to-brain kinetics and retention of plasma proteins and macromolecules occurred concomitant with evidence of heightened state of inflammation (GFAP and COX-2). Indeed, the correlation analyses showed an association between inflammation and parenchymal IgG and apoB abundance. In addition, our recent study showed the colocalization of parenchymal IgG extravasation and increased GFAP abundance in the same SFA-fed mouse model [12]. Interestingly, provision of the anti-oxidants GEA, ALA, niacin, or NA showed similar effects and completely suppressed SFA- induced $\mathrm{BBB}$ dysfunction. The prevention of $\mathrm{BBB}$ disruption by the indicated nutraceuticals occurred concomitant with the suppression of inflammation (GFAP and COX-2) and restoration of plasma total antioxidative status.

Earlier studies suggested that dyslipidemia might be central to brain capillary disturbances in this established model of BBB dysfunction $[9,39,40]$. In the present study, SFA feeding for nine months had no significant effect on the plasma concentration of cholesterol, triglycerides, or NEFA. Cell culture studies suggest several mechanisms by which dietary cholesterol may be pro- 

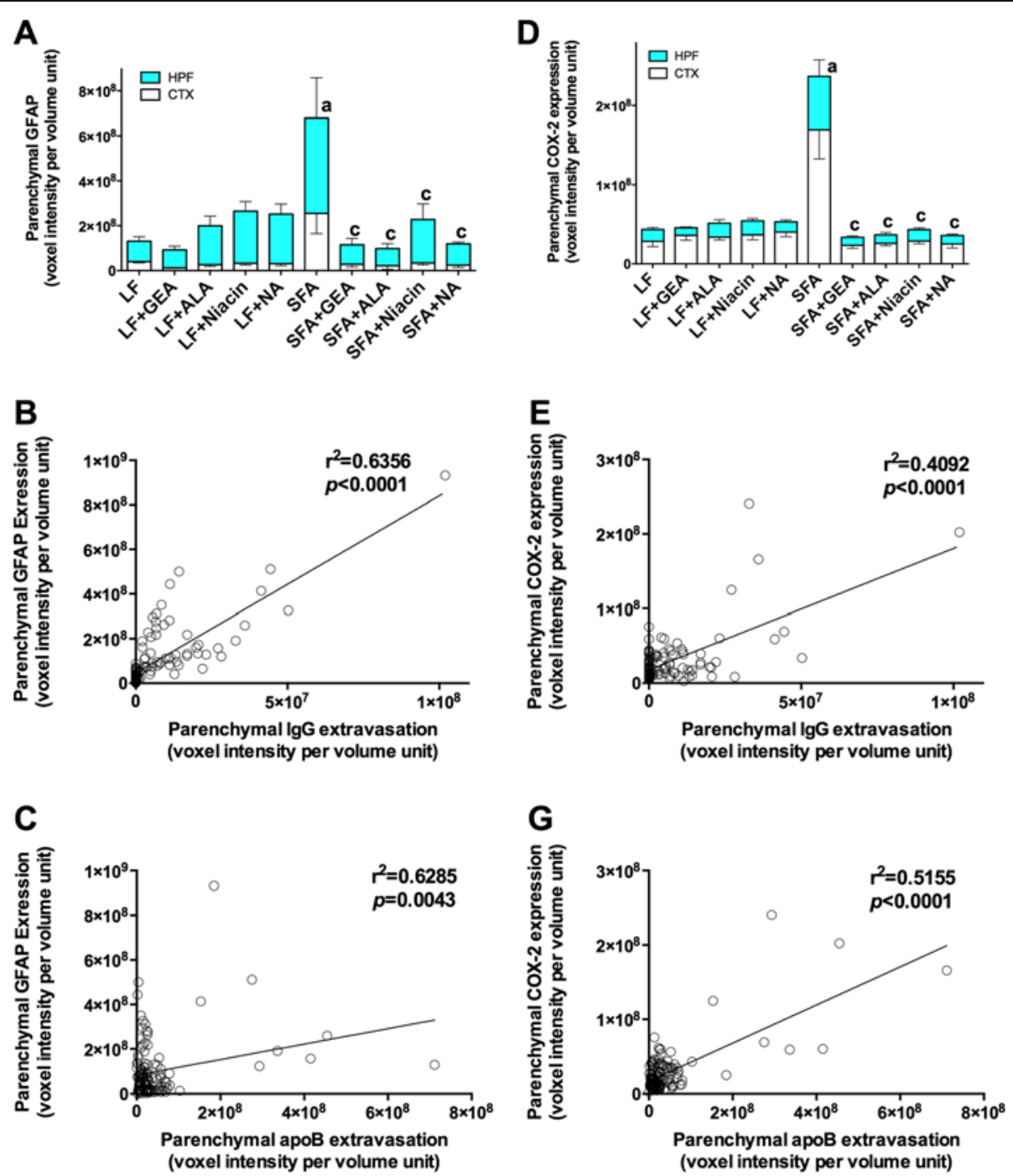

Figure 5 3-D quantitative analyses of cerebral GFAP and COX-2 expressions. The optical staining intensities of GFAP (A) and COX-2 (D) were measured with 3-D image analysis software and shown in the cortex (CTX) and hippocampal formation (HPF) of mice maintained on lowfat control chow (LF), diet enriched in saturated fat (SFA) or each diet supplemented with nutraceutical garlic extract-aged (+GEA), alpha-lipoic acid (+ALA), niacin (+Niacin) or nicotinamide (+NA) for nine months. One-way ANOVA followed by Tukey's post hoc test was used to analyze the statistical significance at $P<0.05$. Data were analyzed in the cortex and hippocampal formation separately, however they are shown as combined since the statistical significance was identical. (a) Significant difference between LF and SFA; (c) Significant difference between SFA and each nutraceutical treatment. (B and E) show the correlation between parenchymal lgG extravasation and GFAP or COX-2 expression in all groups. Pearson's correlation coefficient is indicated. For GFAP and parenchymal abundance of plasma proteins (B and $\mathbf{E}$ ) and for COX-2 versus IgG and apoB (C and $\mathbf{G})$

inflammatory and some of these appear to be analogous to the effects of dietary SFA. Yao and colleagues reported that excess cholesterol causes ER and mitochondrial stress that can lead to apoptosis [41]. Central to the latter, may be regulatory processes via altered ceramide/ sphingosine-1-phosphate homeostasis [42]. Interestingly, Myriocin, an inhibitor of ceramide biosynthesis was effective in ameliorating ceramide accumulation in animals fed diets enriched in SFA [43,44]. Other mechanisms for SFA-induced alterations in the BBB functions include stimulation of NADPH-oxidase derived reactive oxygen species generated by activated microglial cells [45]. Substantially increased parenchymal COX-2 activity and diminished anti-oxidant status are consistent with exaggerated oxidative metabolism. COX-2 is an inducible enzyme most commonly associated with activated macrophages [46]. Increased abundance of COX-2 in SFA mice is consistent with the pro-inflammatory effects of SFA reported in many other studies $[47,48]$. Whilst not directly explored in this study, COX-2 may be associated with activated glial cells in mice fed SFA. Moreover, GFAP, a hallmark feature of activated glial cells, 
A

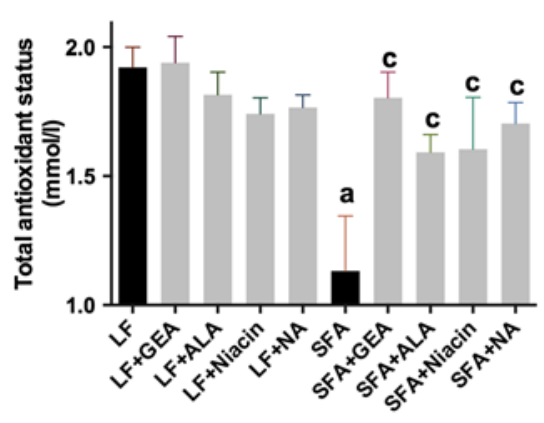

B

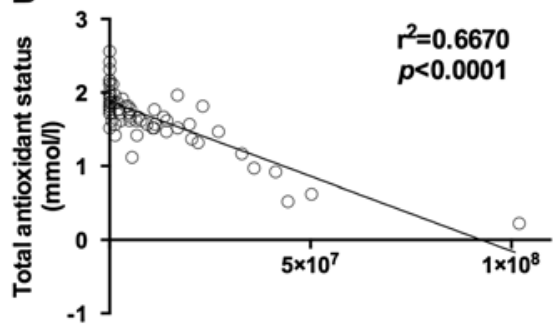

Parenchymal lgG extravasation (voxel intensity per volume unit)

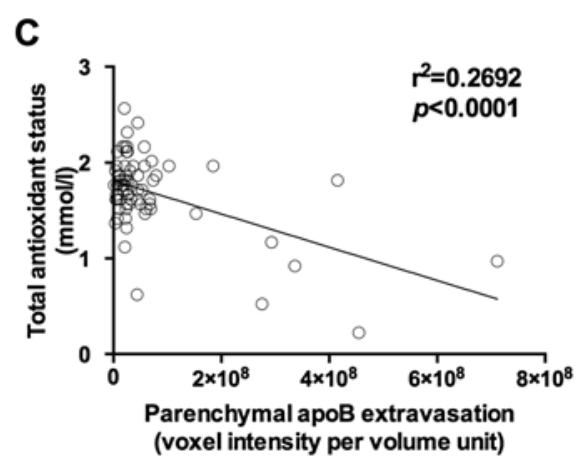

D

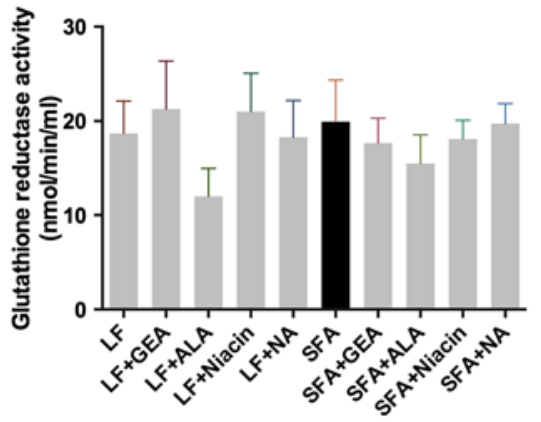

E
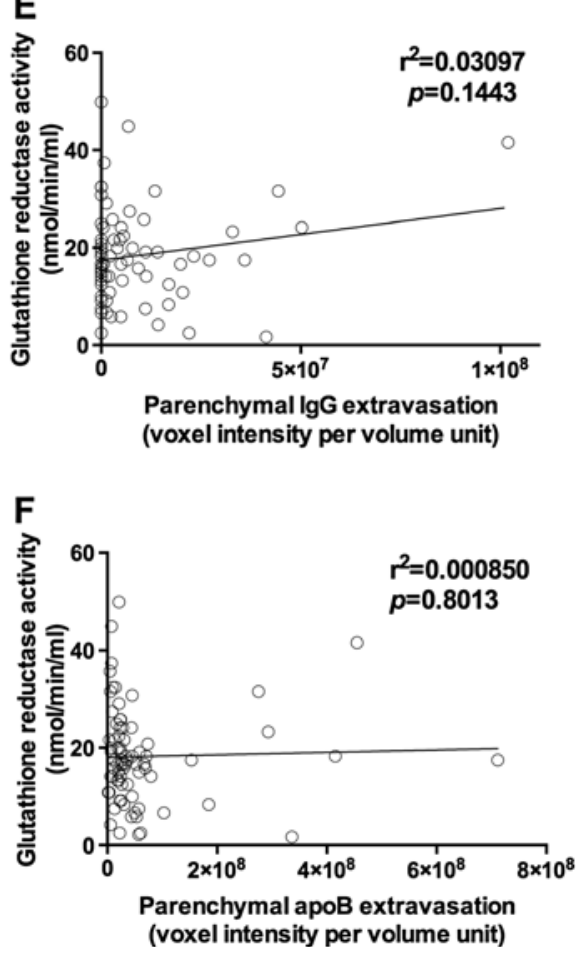

Figure 6 Plasma anti-oxidant status. The plasma total anti-oxidant status (A) and activity of glutathione reductase (D) were measured with commercially available colorimetric kits in mice maintained on low-fat control chow (LF), diet enriched in saturated fat (SFA), or each diet supplemented with nutraceutical garlic extract-aged (+GEA), alpha-lipoic acid (+ALA), niacin (+Niacin), or nicotinamide (+NA) for nine months. One-way ANOVA followed by Tukey's post hoc test was used to analyze the statistical significance at $P<0.05$. (a) Significant difference between LF and SFA; (c) Significant difference between SFA and each nutraceutical treatment. (B and $\mathbf{E}$ ) show the correlation between parenchymal IgG extravasation and total anti-oxidative status or glutathione reductase activity in all groups. The correlation coefficient was analyzed by Pearson's analysis. Similarly, (C and F) show Pearson's correlation coefficient between parenchymal apoB extravasation and total anti-oxidative status or glutathione reductase activity in all groups.

was markedly greater in the SFA treatment group suggesting the heightened cerebrovascular inflammation. In addition, our previous study also showed elevated peri-cerebrovascular CD68 immunoreactivity in SFA-fed mice [39]. Although markers of systemic inflammation were not measured in the present study, glutathione reductase, which serves to recycle glutathione by reducing its oxidized form (glutathione disulfide), was not significantly different in SFA mice, suggesting that this pathway for minimizing oxidative stress was not compromised in SFA-fed mice [49]. Many studies provide evidence that redox (reduction-oxidation) homeostasis is associated with vascular integrity via an axis of exposure to potentially toxic peroxides and free radicals [50-52]. Relevant to the findings described, a study by Kumar et al. reported that elevated plasma malondialdehyde and nitrate/nitrite were positively associated with increased $\mathrm{BBB}$ permeability in subjects with perinatal asphyxia [50]. A recent study reported that a highfat diet (58\% fat of total energy) significantly increased 
BBB permeability concomitant with compromised antioxidative status [7].

A clinical study by Williams et al. showed that GEA improved endothelial function in subjects with cardiovascular disease without altering plasma lipid homeostasis [53]. In insulin resistant subjects, ALA reduced the systemic oxidative stress and improved vascular endothelial function whilst having no effect on plasma lipids or lipoproteins [54]. ALA is transported through the $\mathrm{BBB}$ and reported to suppress cerebral and peripheral inflammation and oxidative stress [55]. Animal studies report that GEA attenuates systemic and central inflammation through increased hemoxygenase-1 activity and superoxide scavenging, and through decreased superoxide production both in vivo and in vitro [56,57]. In rats with subarachnoid hemorrhage, ALA supplementation completely suppressed the BBB disturbances, reduced parenchymal neuroinflammation, and increased plasma anti-oxidative status [58]. In three alternate animal models of systemic inflammation, ALA was shown to reduce cerebral reactive oxygen species and GFAP expression via increased cerebral superoxide dismutase, glutathione, and glutathione peroxidase activity [59-61]. Collectively, the studies suggest that GEA and ALA are likely to protect BBB function by suppressing systemic and central oxidative stress and inflammatory pathways.

In this study, supplementation of niacin or NA significantly reduced plasma cholesterol concentration in SFAfed mice. These two agents also reduced the plasma concentration of NEFA in the LF-control group. Maintenance of BBB function in mice given niacin or NA may have in part reflected diminished vascular exposure to plasma lipids; however, this is unlikely given that the SFA fed group of mice were normolipidemic. Both niacin and NA at the dose rates indicated were equipotent to GEA and ALA in suppressing cerebral neuroinflammation (GFAP and COX-2) and oxidative homeostasis (plasma oxidative status). Ganji et al. showed in vascular endothelial cells in vitro, that niacin significantly increased cellular NADPH and glutathione levels, and reduced reactive oxygen species production and low-density lipoprotein oxidation [62].

A modest increase in cerebral extravasation of plasma IgG and apoB was observed in the LF-treatment group at nine months. The findings are consistent with an aging effect on BBB function recently reported in the same model, commencing at 30 weeks of age [12]. We extend on those findings and show that the supplementation of anti-oxidative nutraceutical agents did not prevent these aging-related alterations to BBB function. This finding is perhaps not unexpected in the context that TAS was similar to the LF control group. The findings suggest that the aging-related alterations in BBB function may occur independent of oxidative stress pathways [63-65].

\section{Conclusions}

The primary findings of this study showed that compromised systemic anti-oxidative status induced by chronic SFA diet ingestion was associated with BBB dysfunction and neurovascular inflammatory responses. The provision of the anti-oxidants GEA, ALA, niacin, and NA prevents disruption of the BBB in high SFA-fed mice, concomitant with an improved redox state. However, the indicated nutraceuticals had no beneficial effects on aging-related disturbance in BBB function.

\begin{abstract}
Abbreviations
ALA: Alpha lipoic acid; apoB: Apolipoprotein B; BBB: Blood-brain barrier; COX-2: Cyclooxygenase-2; GEA: Garlic extracts-aged; GFAP: Glial fibrillar acidic protein; IgG: Immunoglobulin G; LF: Low-fat; NA: Nicotinamide; NEFA: Nonesterified fatty acids; SFA: Saturated fatty acids; TAS: Total anti-oxidant status.
\end{abstract}

\section{Competing interests}

The authors declare that they have no competing interests.

\section{Authors' contributions}

RT conceived and designed the study and was involved in sample and data collection, data interpretation, and manuscript preparation. MMPG, VL, and CG were involved in sample and data collection. JCM was responsible for the supervision of the entire project and was involved in the study design, data interpretation, manuscript preparation, and funding. All authors read and approved the final manuscript.

\section{Author's information}

RT is a Research Fellow of the National Health and Medical Research Council of Australia and of School of Public Health, Faculty of Health Sciences, Curtin University. MMPG, VL, and CG are PhD candidates of the School of Public Health, Faculty of Health Sciences, Curtin University, supervised by RT and JCM. MMPG, VL, and CG are supported by the Australian Postgraduate Awards scholarship. JCM is a Professor of Faculty of Health Sciences, Curtin University, and a Director of Centre for Metabolic Fitness, Australian Technology Network.

\section{Acknowledgements}

The authors would like to acknowledge Professor Satvinder Dhaliwal (Biostatistician, School of Public Health, Curtin University) for his assistance in statistical analyses. This project was financially supported by an NHMRC Project Grant.

Received: 21 December 2012 Accepted: 8 June 2013

Published: 19 June 2013

\section{References}

1. Zlokovic BV: The blood-brain barrier in health and chronic neurodegenerative disorders. Neuron 2008, 57:178-201.

2. Kalaria RN: Vascular basis for brain degeneration: faltering controls and risk factors for dementia. Nutr Rev 2010, 68(Suppl 2):S74-S87.

3. Dickstein DL, Walsh J, Brautigam H, Stockton SD Jr, Gandy S, Hof PR: Role of vascular risk factors and vascular dysfunction in Alzheimer's disease. Mt Sinai J Med N Y 2010, 77:82-102.

4. Miyakawa T: Vascular pathology in Alzheimer's disease. Psychogeriat 2010, 10:39-44.

5. Fisher CM: The arterial lesions underlying lacunes. Acta Neuropathol 1968, 12:1-15.

6. Stolp HB, Johansson PA, Habgood MD, Dziegielewska KM, Saunders NR, Ek CJ: Effects of neonatal systemic inflammation on blood-brain barrier permeability and behaviour in juvenile and adult rats. Cardiovasc Psychiatry Neurol 2011, 2011:469046.

7. Nerurkar PV, Johns LM, Buesa LM, Kipyakwai G, Volper E, Sato R, Shah P, Feher D, Williams PG, Nerurkar VR: Momordica charantia (bitter melon) attenuates high-fat diet-associated oxidative stress and neuroinflammation. J Neuroinflam 2011, 8:64. 
8. Drake C, Boutin H, Jones MS, Denes A, McColl BW, Selvarajah JR, Hulme S, Georgiou RF, Hinz R, Gerhard A, Vail A, Prenant C, Julyan P, Maroy R, Brown G, Smigova A, Herholz K, Kassiou M, Crossman D, Francis S, Proctor SD, Russell JC, Hopkins SJ, Tyrrell PJ, Rothwell NJ, Allan SM: Brain inflammation is induced by co-morbidities and risk factors for stroke. Brain Behav Immun 2011, 25:1113-1122.

9. Takechi R, Galloway S, Pallebage-Gamarallage MM, Wellington CL, Johnsen RD, Dhaliwal SS, Mamo JC: Differential effects of dietary fatty acids on the cerebral distribution of plasma-derived apo $B$ lipoproteins with amyloid-beta. Br J Nutr 2010, 103:652-662.

10. Takechi R, Galloway S, Pallebage-Gamarallage MM, Lam V, Mamo JC: Dietary fats, cerebrovasculature integrity and Alzheimer's disease risk. Prog Lipid Res 2010, 49:159-170.

11. Pallebage-Gamarallage MM, Lam V, Takechi R, Galloway S, Mamo JC: A diet enriched in docosahexanoic Acid exacerbates brain parenchymal extravasation of apo B lipoproteins induced by chronic ingestion of saturated fats. Int J Vasc Med 2012, 2012:647689.

12. Takechi R, Pallebage-Gamarallage MM, Lam V, Giles C, Mamo JC: Aging-related changes in blood-brain barrier integrity and the effect of dietary fat. Neurodegen Dis 2012.

13. Takechi R, Galloway S, Pallebage-Gamarallage MM, Mamo JC: Chylomicron amyloid-beta in the aetiology of Alzheimer's disease. Atheroscler Suppl 2008, 9:19-25.

14. Ifergan I, Wosik K, Cayrol R, Kebir H, Auger C, Bernard M, Bouthillier A, Moumdjian R, Duquette $P$, Prat A: Statins reduce human blood-brain barrier permeability and restrict leukocyte migration: relevance to multiple sclerosis. Ann Neurol 2006, 60:45-55.

15. Kalayci R, Kaya M, Elmas I, Arican N, Ahishali B, Uzun H, Bilgic B, Kucuk M, Kudat H: Effects of atorvastatin on blood-brain barrier permeability during L-NAME hypertension followed by angiotensin-II in rats. Brain Res 2005, 1042:184-193.

16. Pallebage-Gamarallage MM, Takechi R, Lam V, Galloway S, Dhaliwal S, Mamo JC: Post-prandial lipid metabolism, lipid-modulating agents and cerebrovascular integrity: implications for dementia risk. Atheroscler Suppl 2010, 11:49-54.

17. Takechi R, Galloway S, Pallebage-Gamarallage MM, Lam V, Dhaliwal SS, Mamo JC: Probucol prevents blood-brain barrier dysfunction in wild-type mice induced by saturated fat or cholesterol feeding. Clin Exp Pharmacol Physiol 2013, 40:45-52.

18. Pallebage-Gamarallage M, Lam V, Takechi R, Galloway S, Clark K, Mamo J. Restoration of dietary-fat induced blood-brain barrier dysfunction by anti-inflammatory lipid-modulating agents. Lipids Health Dis 2012, 11:117.

19. Chen X, Ghribi O, Geiger JD: Caffeine protects against disruptions of the blood-brain barrier in animal models of Alzheimer's and Parkinson's diseases. J Alzheimer Dis 2010, 20(Suppl 1):S127-S141.

20. Chen X, Gawryluk JW, Wagener JF, Ghribi O, Geiger JD: Caffeine blocks disruption of blood brain barrier in a rabbit model of Alzheimer's disease. J Neuroinflam 2008, 5:12.

21. Mustata GT, Rosca M, Biemel KM, Reihl O, Smith MA, Viswanathan A, Strauch C, Du Y, Tang J, Kern TS, Lederer MO, Brownlee M, Weiss MF, Monnier VM: Paradoxical effects of green tea (Camellia sinensis) and antioxidant vitamins in diabetic rats: improved retinopathy and renal mitochondrial defects but deterioration of collagen matrix glycoxidation and cross-linking. Diabetes 2005, 54:517-526.

22. Patel RP, Boersma BJ, Crawford JH, Hogg N, Kirk M, Kalyanaraman B, Parks DA, Barnes S, Darley-Usmar V: Antioxidant mechanisms of isoflavones in lipid systems: paradoxical effects of peroxyl radical scavenging. Free Radic Biol Med 2001, 31:1570-1581.

23. Geng Z, Rong Y, Lau BH: S-allyl cysteine inhibits activation of nuclear factor kappa B in human T cells. Free Radic Biol Med 1997, 23:345-350.

24. Kim HK, Choi YW, Lee EN, Park JK, Kim SG, Park DJ, Kim BS, Lim YT, Yoon S: 5-Hydroxymethylfurfural from black garlic extract prevents TNF alpha-induced monocytic cell adhesion to HUVECs by suppression of vascular cell adhesion molecule- 1 expression, reactive oxygen species generation and NF-kappaB activation. Phytother Res 2011, 25:965-974.

25. Ban JO, Oh JH, Kim TM, Kim DJ, Jeong HS, Han SB, Hong JT: Anti-inflammatory and arthritic effects of thiacremonone, a novel sulfur compound isolated from garlic via inhibition of NF-kappaB. Arthritis Res Ther 2009, 11:R145.

26. Rojas P, Serrano-Garcia N, Medina-Campos ON, Pedraza-Chaverri J, Maldonado PD, Ruiz-Sanchez E: S-Allylcysteine, a garlic compound, protects against oxidative stress in 1-methyl-4-phenylpyridiniuminduced parkinsonism in mice. J Nutr Biochem 2011, 22:937-944.

27. Chauhan NB, Sandoval J: Amelioration of early cognitive deficits by aged garlic extract in Alzheimer's transgenic mice. Phytother Res 2007, 21:629-640.

28. Chauhan NB: Effect of aged garlic extract on APP processing and tau phosphorylation in Alzheimer's transgenic model Tg2576. J Ethnopharmacol 2006, 108:385-394.

29. Pasinetti GM, Eberstein JA: Metabolic syndrome and the role of dietary lifestyles in Alzheimer's disease. J Neurochem 2008, 106:1503-1514.

30. Ray B, Chauhan NB, Lahiri DK: Oxidative insults to neurons and synapse are prevented by aged garlic extract and S-allyl-L-cysteine treatment in the neuronal culture and APP-Tg mouse model. J Neurochem 2011, 117:388-402.

31. Gupta VB, Indi SS, Rao KS: Garlic extract exhibits antiamyloidogenic activity on amyloid-beta fibrillogenesis: relevance to Alzheimer's disease. Phytother Res 2009, 23:111-115.

32. Maczurek A, Hager K, Kenklies M, Sharman M, Martins R, Engel J, Carlson DA, Munch G: Lipoic acid as an anti-inflammatory and neuroprotective treatment for Alzheimer's disease. Adv Drug Deliv Rev 2008, 60:1463-1470.

33. Toklu HZ, Hakan T, Biber N, Solakoglu S, Ogunc AV, Sener G: The protective effect of alpha lipoic acid against traumatic brain injury in rats. Free Rad Res 2009, 43:658-667.

34. MacKay D, Hathcock J, Guarneri E: Niacin: chemical forms, bioavailability, and health effects. Nutr Rev 2012, 70:357-366.

35. Morris MC, Evans DA, Bienias JL, Scherr PA, Tangney CC, Hebert LE, Bennett DA, Wilson RS, Aggarwal N: Dietary niacin and the risk of incident Alzheimer's disease and of cognitive decline. J Neurol Neurosurg Psychiatry 2004, 75:1093-1099.

36. Lawson LD, Gardner CD: Composition, stability, and bioavailability of garlic products used in a clinical trial. J Agric Food Chem 2005, 53:6254-6261.

37. Van Broeck B, Van Broeckhoven C, Kumar-Singh S: Current insights into molecular mechanisms of Alzheimer disease and their implications for therapeutic approaches. Neurodegener Dis 2007, 4:349-365.

38. Kumar-Singh S, Pirici D, McGowan E, Serneels S, Ceuterick C, Hardy J, Duff K, Dickson D, Van Broeckhoven C: Dense-core plaques in Tg2576 and PSAPP mouse models of Alzheimer's disease are centered on vessel walls. Am J Pathol 2005, 167:527-543.

39. Bowman GL, Kaye JA, Quinn JF: Dyslipidemia and blood-brain barrier integrity in Alzheimer's disease. Curr Gerontol Geriatr Res 2012, 2012:184042.

40. Burgess BL, Mclsaac SA, Naus KE, Chan JY, Tansley GH, Yang J, Miao F, Ross CJ, van Eck M, Hayden MR, van Nostrand W, St George-Hyslop P, Westaway $D$, Wellington $C$ : Elevated plasma triglyceride levels precede amyloid deposition in Alzheimer's disease mouse models with abundant A beta in plasma. Neurobiol Dis 2006, 24:114-127.

41. Yao PM, Tabas I: Free cholesterol loading of macrophages is associated with widespread mitochondrial dysfunction and activation of the mitochondrial apoptosis pathway. J Biol Chem 2001, 276:42468-42476.

42. Messmer TO, Wang E, Stevens VL, Merrill AH Jr: Sphingolipid biosynthesis by rat liver cells: effects of serine, fatty acids and lipoproteins. J Nutr 1989, 119:534-538.

43. Ussher JR, Koves TR, Cadete VJ, Zhang L, Jaswal JS, Swyrd SJ, Lopaschuk DG, Proctor SD, Keung W, Muoio DM, Lopaschuk GD: Inhibition of de novo ceramide synthesis reverses diet-induced insulin resistance and enhances whole-body oxygen consumption. Diabetes 2010, 59:2453-2464.

44. Holland WL, Brozinick JT, Wang LP, Hawkins ED, Sargent KM, Liu Y, Narra K, Hoehn KL, Knotts TA, Siesky A, Nelson DH, Karathanasis SK, Fontenot GK, Birnbaum MJ, Summers S: Inhibition of ceramide synthesis ameliorates glucocorticoid-, saturated-fat-, and obesity-induced insulin resistance. Cell Metab 2007, 5:167-179.

45. Choi SH, Aid S, Kim HW, Jackson SH, Bosetti F: Inhibition of NADPH oxidase promotes alternative and anti-inflammatory microglial activation during neuroinflammation. J Neurochem 2012, 120:292-301.

46. Tzeng SF, Hsiao HY, Mak OT: Prostaglandins and cyclooxygenases in glial cells during brain inflammation. Curr Drug Targets Inflamm Allergy 2005 4:335-340.

47. Kadotani A, Tsuchiya Y, Hatakeyama H, Katagiri H, Kanzaki M: Different impacts of saturated and unsaturated free fatty acids on COX-2 expression in C(2)C(12) myotubes. Am J Physiol Endocrinol Metab 2009, 297:E1291-E1303. 
48. Lee JY, Sohn KH, Rhee SH, Hwang D: Saturated fatty acids, but not unsaturated fatty acids, induce the expression of cyclooxygenase-2 mediated through Toll-like receptor 4. J Biol Chem 2001, 276:16683-16689.

49. Meister A: Glutathione metabolism and its selective modification. J Biol Chem 1988, 263:17205-17208

50. Kumar A, Mittal R, Khanna HD, Basu S: Free radical injury and blood-brain barrier permeability in hypoxic-ischemic encephalopathy. Pediatrics 2008, 122:e722-e727.

51. Lehner C, Gehwolf R, Tempfer H, Krizbai I, Hennig B, Bauer HC, Bauer H: Oxidative stress and blood-brain barrier dysfunction under particular consideration of matrix metalloproteinases. Antioxid Redox Signal 2011, 15:1305-1323.

52. Potenza MA, Gagliardi S, De Benedictis L, Zigrino A, Tiravanti E, Colantuono G, Federici A, Lorusso L, Benagiano V, Quon MJ, Montagnani M: Treatment of spontaneously hypertensive rats with rosiglitazone ameliorates cardiovascular pathophysiology via antioxidant mechanisms in the vasculature. Am J Physiol Endocrinol Metab 2009, 297:E685-E694.

53. Williams MJ, Sutherland WH, McCormick MP, Yeoman DJ, de Jong SA: Aged garlic extract improves endothelial function in men with coronary artery disease. Phytother Res 2005, 19:314-319.

54. Xiang G, Pu J, Yue L, Hou J, Sun H: Alpha-lipoic acid can improve endothelial dysfunction in subjects with impaired fasting glucose. Metabolism 2011, 60:480-485.

55. Chng HT, New LS, Neo AH, Goh CW, Browne ER, Chan EC: Distribution study of orally administered lipoic acid in rat brain tissues. Brain Res 2009, 1251:80-86.

56. Park HJ, Jeon BT, Kim HC, Roh GS, Shin JH, Sung NJ, Han J, Kang D: Aged red garlic extract reduces lipopolysaccharide-induced nitric oxide production in RAW 264.7 macrophages and acute pulmonary inflammation through haeme oxygenase-1 induction. Acta Physiol (Oxf) 2012, 205:61-70.

57. Morihara N, Hayama M, Fujii H: Aged garlic extract scavenges superoxide radicals. Plant Foods Hum Nutr 2011, 66:17-21.

58. Ersahin $M$, Toklu HZ, Cetinel S, Yuksel M, Erzik C, Berkman MZ, Yegen BC Sener G: Alpha lipoic acid alleviates oxidative stress and preserves blood brain permeability in rats with subarachnoid hemorrhage. Neurochem Res 2010, 35:418-428.

59. Martins RR, de Oliveira Macedo UB, Leite LD, Rezende AA, Brandao-Neto J, Almeida M: Lipoic acid and moderate swimming improves the estrous cycle and oxidative stress in Wistar rats. Appl Physiol Nutr Metab 2011, 36:693-697.

60. Jesudason EP, Masilamoni JG, Jebaraj CE, Paul SF, Jayakumar R: Efficacy of DL-alpha lipoic acid against systemic inflammation-induced mice: antioxidant defense system. Mol Cell Biochem 2008, 313:113-123.

61. Skibska B, Jozefowicz-Okonkwo G, Goraca A: Protective effects of early administration of alpha-lipoic acid against lipopolysaccharide-induced plasma lipid peroxidation. Pharmacol Rep 2006, 58:399-404.

62. Ganji SH, Qin S, Zhang L, Kamanna VS, Kashyap ML: Niacin inhibits vascular oxidative stress, redox-sensitive genes, and monocyte adhesion to human aortic endothelial cells. Atherosclerosis 2009, 202:68-75.

63. Black JE, Polinsky M, Greenough WT: Progressive failure of cerebral angiogenesis supporting neural plasticity in aging rats. Neurobiol Aging 1989, 10:353-358.

64. Chavez JC, LaManna JC: Hypoxia-inducible factor-1alpha accumulation in the rat brain in response to hypoxia and ischemia is attenuated during aging. Ad Exp Med Biol 2003, 510:337-341.

65. Rapino C, Bianchi G, Di Giulio C, Centurione L, Cacchio M, Antonucci A, Cataldi A: HIF-1alpha cytoplasmic accumulation is associated with cell death in old rat cerebral cortex exposed to intermittent hypoxia. Aging Cell 2005, 4:177-185.

doi:10.1186/1742-2094-10-73

Cite this article as: Takechi et al:: Nutraceutical agents with antiinflammatory properties prevent dietary saturated-fat induced disturbances in blood-brain barrier function in wild-type mice. Journal of Neuroinflammation 2013 10:73.

\section{Submit your next manuscript to BioMed Central and take full advantage of:}

- Convenient online submission

- Thorough peer review

- No space constraints or color figure charges

- Immediate publication on acceptance

- Inclusion in PubMed, CAS, Scopus and Google Scholar

- Research which is freely available for redistribution

Submit your manuscript at www.biomedcentral.com/submit
( Biomed Central 